\title{
Serum Adipocyte Fatty Acid-Binding Protein 4 Levels Are Independently Associated with Radioisotope Glomerular Filtration Rate in Type 2 Diabetic Patients with Early Diabetic Nephropathy
}

\author{
Xiaoqing Ni, ${ }^{1}$ Yunjuan Gu $\left(\mathbb{D},{ }^{2}\right.$ Haoyong Yu, ${ }^{3}$ Shenqi Wang ${ }^{\circ},{ }^{3}$ Ying Chen, \\ Xinlei Wang, ${ }^{2}$ Xinlu Yuan, ${ }^{2}$ and Weiping Jia ${ }^{3}{ }^{3}$ \\ ${ }^{1}$ Department of Geriatrics, Affiliated Hospital of Nantong University, Jiangsu, China \\ ${ }^{2}$ Department of Endocrinology and Metabolism, Affiliated Hospital of Nantong University, Jiangsu, China \\ ${ }^{3}$ Department of Endocrinology and Metabolism, Shanghai Jiao Tong University Affiliated Sixth People's Hospital, \\ Shanghai Diabetes Institute, Shanghai Key Laboratory of Diabetes Mellitus, Shanghai Clinical Center for Diabetes, Shanghai, China \\ Correspondence should be addressed to Yunjuan Gu; desette@ntu.edu.cn and Weiping Jia; wpjia@yahoo.com
}

Received 28 August 2017; Revised 17 January 2018; Accepted 21 January 2018; Published 27 May 2018

Academic Editor: Himanshu Garg

Copyright (C) 2018 Xiaoqing Ni et al. This is an open access article distributed under the Creative Commons Attribution License, which permits unrestricted use, distribution, and reproduction in any medium, provided the original work is properly cited.

\begin{abstract}
Serum fatty acid-binding protein 4 (FABP4) has been linked to renal dysfunction. This study evaluated the association between serum FABP4 and the radioisotope glomerular filtration rate (rGFR) in type 2 diabetic patients (T2DM) with early diabetic nephropathy. Twenty healthy controls and 172 patients with T2DM were enrolled. Serum FABP4 and renal impairment biomarkers including urinary albumin-to-creatinine ratio (UACR), serum retinal-binding protein 4 (RBP4), urinary cystatin C-to-creatinine ratio $(\mathrm{CysC} / \mathrm{Cr})$, and neutrophil gelatinase-associated lipocalin-to-creatinine ratio (NGAL/Cr) were measured. Diethylenetriaminepentaacetic acid (99mTc-DTPA) was used to test rGFR. Serum FABP4 levels were higher in T2DM patients compared with the controls. There was no significant correlation between serum FABP4 and UACR in patients with T2DM. Multivariate stepwise regression analysis showed that, in patients with T2DM, FABP4 was significantly associated with rGFR while CysC/Cr and RBP4 were significantly associated with UACR independently. But UACR had no independent association with rGFR. NGAL/Cr had no significant correlation with either rGFR or UACR. FABP4 might be an early biomarker for diabetic nephropathy if combined with UACR.
\end{abstract}

\section{Introduction}

Diabetic nephropathy (DN) is a chronic complication of diabetes, characterized by the presence of urinary albumin excretion and/or accompanied by a gradual deterioration in the glomerular filtration rate (GFR). It affects approximately $20-40 \%$ of patients with diabetes mellitus and is recognized as the leading cause of chronic kidney disease (CKD) and end-stage renal disease $[1,2]$. Patients with CKD, irrespective of etiology, are at high risk for cardiovascular disease and mortality [3]. A systematic review reported that patients with type 2 diabetes mellitus (T2DM) who underwent intensive glycemic control and lipid interventions did not show improvement in clinical outcomes including all-cause mortality and death from cardiovascular causes, incident kidney failure, and nonfatal cardiovascular events [4]. Although the pathogenesis of DN remains unclear, evidence indicates that early recognition and intervention of $\mathrm{DN}$ may delay the progression to end-stage renal disease and cardiovascular disease [5]. However, diagnosis of DN is often delayed since the symptoms are usually insidious and develop slowly.

Considering the significant effect of CKD, Kidney Disease Outcomes Quality Initiative (KDOQI) and Kidney Disease Improving Global Outcomes (KDIGO) recommended a focal point of early identification of $\mathrm{CKD}[2,6]$. According to KDIGO recommendation, GFR (estimated GFR, eGFR) and 
albuminuria (urine albumin-to-creatinine ratio, UACR) are the main indicators used for initial detection and staging of acute and chronic kidney disease in adults [7, 8]. GFR is a well-validated evaluation index for kidney function and albuminuria is a marker of kidney damage [8]. GFR can be measured directly using a clearance procedure or by using equations. However, the estimating equations are relatively imprecise, with approximately $10-20 \%$ of estimates deviating by more than $30 \%$ from the measured GFR [8]. Compared with eGFR, radioisotope GFR (rGFR) using diethylenetriaminepentaacetic acid (Tc-99 m DTPA), a radio-labeled pharmaceutical agent, is a more precise measurement in clinic trials [9]. And as for UACR, it was reported to have a continuous association with the risk for progression of $\mathrm{CKD}$ to end-stage renal disease [10] and was recommended for the detection and staging of kidney injury [8]. But in some clinical conditions, it may remain within the normal range in early-stage DN whereas the GFR probably has already decreased [11]. It is therefore necessary to identify biomarkers that are more accurate, sensitive, and clinically attainable to reflect early renal impairment in DN. Additionally, such proposed biomarkers might be valuable for large-scale programs for early screening and prediction of the prognosis of $\mathrm{DN}$.

In the past decades, several biomarkers have emerged for detection of early DN besides GFR and UACR. Among them, adipocyte fatty acid-binding protein (FABP) 4 has attracted increased attention. As a member of the calycin protein superfamily, it is a small intracellular protein expressed mainly in adipose tissue and by macrophages. Its physiological role includes fatty acid storage, transportation, metabolism, and the regulation of cell proliferation and differentiation. A high circulating FABP4 concentration was reported to correlate with body weight, glucose and lipid metabolism, and atherosclerosis and was considered an early risk factor for the progression of metabolic syndrome [1217]. FABP4 was also reported at increased concentrations in nondiabetic as well as T2DM patients with end-stage renal disease $[18,19]$. Yeung et al. reported that serum level of adipocyte FABP had a significantly inverse relationship with eGFR and was independently associated with macrovascular complications and DN staging classified by albuminuria [20].

Another adipokine, serum retinol-binding protein 4 (RBP4) is a small protein synthesized mainly in the liver and adipocytes and belongs to the lipocalin family [21]. Several studies suggest that upregulation of RBP4 correlates with obesity, insulin resistance, renal dysfunction, and cardiovascular disease in patients with T2DM [21-24]. Murata et al. reported that, in 149 T2DM patients, eGFR was an independent determinant for increased serum RBP4 levels [25].

Neutrophil gelatinase-associated lipocalin (NGAL), which is produced by epithelial cells and neutrophils, has recently gained increased attention as a sensitive and specific biomarker of tubular damage [26, 27]. Nielsen et al. investigated 177 patients with T2DM and found that after a 5-year follow-up, a high level of baseline urinary NGAL was negatively associated with GFR and positively associated with the progression to macroalbuminuria [28]. Another study reported that, in T2DM patients of short duration, urinary NGAL levels may be more sensitive than UACR for monitoring DN in the early stages [29]. A recent study reported that, in T2DM patients, both serum RBP4 and NGAL concentrations significantly and positively correlated with UACR and negatively correlated with eGFR [30].

Cystatin C (CysC), a cysteine proteinase inhibitor produced by most nucleated cells [31], has been considered as a replacement for serum creatinine or even as an alternative endogenous marker for GFR $[32,33]$. The plasma concentration of CysC is stable, since it can be freely filtered through the glomerular membrane and reabsorbed and catabolized by renal tubular cells [34-36]. A large number of studies have reported that plasma levels of CysC, or eGFR formulas based on CysC, were good markers of early renal dysfunction in patients with diabetes [37-39]. However, recent studies also report that using urinary $\mathrm{CysC}$ or the $\mathrm{CysC}$-to-creatinine ratio $(\mathrm{Cys} / \mathrm{Cr})$ to estimate renal impairment is less convincing $[40,41]$.

Focused on rGFR as the reference in the early stages of diabetic nephropathy, the aim of the current study was to evaluate the association among renal impairment biomarkers, with rGFR, and UACR in patients with T2DM to identity a sensitive predictor for early DN.

\section{Patients and Methods}

2.1. Study Population. From June 2010 to January 2013, a total of 172 T2DM inpatients were enrolled at the Department of Endocrinology and Metabolism at Shanghai Jiao Tong University Affiliated Sixth People's Hospital. T2DM patients were diagnosed according to World Health Organization diagnostic criteria (1999) [42]. Patients with type 1 diabetes mellitus or secondary diabetes, malignancy, chronic liver disorders, chronic or acute inflammation, morbid obesity (body mass index $(\mathrm{BMI}) \geq 40 \mathrm{~kg} / \mathrm{m}^{2}$ ), familial hypercholesterolemia, or $\mathrm{rGFR}<30 \mathrm{~mL} / \mathrm{min} / 1.73 \mathrm{~m}^{2}$ were excluded. Urine and serum samples were collected on the second morning of hospitalization after a 10 -h overnight fast. Twenty healthy controls were selected from the epidemiological survey database of the Shanghai Caoyang community who were matched for race, ethnicity, age, gender, and BMI with the T2DM patients in this study.

The study was approved by the Shanghai Jiao Tong University Affiliated Sixth People's Hospital Ethics Committee and was conducted in accordance with the principles contained within the Declaration of Helsinki. Each patient provided written informed consent.

Information on sex, age, anthropometric parameters including height, weight, waist circumference (WC), and blood pressure was collected. BMI was calculated as weight (kg) divided by the square of height $\left(\mathrm{m}^{2}\right)$. Venous blood samples were collected by venipuncture into vacuum tubes. Serum samples were separated within $30 \mathrm{~min}$ after blood sample collection, centrifuged at $3500 \times \mathrm{g}$ for $10 \mathrm{~min}$, and stored at $-20^{\circ} \mathrm{C}$.

2.2. Laboratory Measurements. Fasting plasma glucose, 2$\mathrm{h}$ postprandial plasma glucose, total cholesterol, triglycerides, high-density lipoprotein cholesterol, and low-density 
lipoprotein cholesterol were tested by enzymatic procedures using an autoanalyzer (Hitachi 7600-020; Hitachi, Tokyo, Japan). Glycosylated hemoglobin Alc values were measured by high-performance liquid chromatography (BioRad Laboratories, Hercules, CA). Serum RBP4 was measured with a radioimmunoassay kit (Phoenix, Belmont, CA). Serum high-sensitivity C-reactive protein (hsCRP) was measured using a particle-enhanced immunoturbidimetric assay (Dade Behring Inc., Newark, NJ). Serum FABP4, CysC, and NGAL were measured using a sandwich enzyme-linked immunosorbent assay (BioVendor Laboratory Medicine, Modrice, Czech Republic). Urinary albumin and creatinine levels were measured using the first morning void urine samples by immunonephelometry and a BN II analyzer (Siemens Diagnostics). UACR was calculated by dividing the urinary albumin concentration by the urine creatinine concentration. Urine CysC/Cr ratio and NGAL-to-creatinine ratio (NGAL/Cr) were also calculated. The rGFR was directly measured by $99 \mathrm{mTc}$-DTPA.

Diabetic patients were divided into three groups according to UACR level [7]: normal albuminuria group (UACR $<30 \mu \mathrm{g} / \mathrm{mg}$ ); microalbuminuria group $(30 \mu \mathrm{g} / \mathrm{mg} \leq \mathrm{UACR}$ $<300 \mu \mathrm{g} / \mathrm{mg}$ ); and macroalbuminuria group (UACR $\geq$ $300 \mu \mathrm{g} / \mathrm{mg}$ ). According to the rGFR stratum, T2DM patients were also divided into three subgroups [7]: normal renal function $\left(\mathrm{rGFR} \geq 90 \mathrm{~mL} / \mathrm{min} / 1.73 \mathrm{~m}^{2}\right)$; mild renal dysfunction $\left(60 \mathrm{~mL} / \mathrm{min} / 1.73 \mathrm{~m}^{2} \leq \mathrm{rGFR}<90 \mathrm{~mL} / \mathrm{min} / 1.73 \mathrm{~m}^{2}\right)$; and moderate renal dysfunction $\left(30 \mathrm{~mL} / \mathrm{min} / 1.73 \mathrm{~m}^{2} \leq \mathrm{rGFR}<\right.$ $60 \mathrm{~mL} / \mathrm{min} / 1.73 \mathrm{~m}^{2}$ ).

2.3. Statistical Analysis. Continuous variables with a normal distribution are shown as mean \pm standard deviation (SD). Data that did not have a normal distribution determined using the Kolmogorov-Smirnov test were logarithmically transformed before analysis and are shown as median (interquartile range). If data were still not normally distributed after transformation, they were analyzed using the Wilcoxon rank sum test. One-way ANOVA or the $\chi^{2}$ test was used for comparisons between groups, and correlations between variables were adjusted using partial correlation. Stepwise multivariate regression analysis was used to determine correlation of variables with rGFR or UACR as dependent variables. All analyses were performed using SPSS statistical package version 18.0 (SPSS, Chicago, IL, USA). A $P$ value $<0.05$ was considered statistically significant.

\section{Results}

As shown in Table 1, there were no significant differences between T2DM patients and healthy controls in terms of age, smoking status, BMI, lipid profiles, RBP4, rGFR, hsCRP, and CysC/Cr. Patients with T2DM had a significantly larger waist circumference, higher blood pressure, HbAlc, fasting plasma glucose, 2-h plasma glucose, UACR, FABP4, and NGAL/Cr compared with the control group (all $P<0.05$ ). When compared with the control group separately, the macroalbuminuria subgroup had significantly higher levels of RBP4 and hsCRP (both $P<0.05$ ), and microalbuminuria and macroalbuminuria subgroups had significantly lower rGFR (both $P<$ 0.05). Among the three diabetic subgroups, patients with microalbuminuria were relatively older $(P=0.034)$. In the macroalbuminuria subgroup, the number of patients using angiotensin II receptor blockers (ARB)/angiotensin-converting enzyme inhibitors (ACEI) was significantly higher $(P=0.046)$, and systolic blood pressure (SBP), total cholesterol, triglycerides, low-density lipoprotein cholesterol, RBP4, hsCRP, and UACR were significantly higher than the other two subgroups (all $P<0.05$ ). Among the three diabetic subgroups, rGFR levels decreased with increasing UACR (both $P<0.0001$ ). FABP4, NGAL/Cr, and $\mathrm{CysC/Cr}$ were not significantly different among the three subgroups.

Based on rGFR stratum, among the three diabetic subgroups, age, diabetes duration, percentage of hypertension, WC, SBP, RBP4, UACR, and FABP4 all increased with the decrease in rGFR (all $P<0.05$ ) (Table 2). For NGAL/Cr, although there were no significant differences among the three subgroups, when compared with the control group, NGAL/Cr increased in the two subgroups with rGFR less than $90 \mathrm{~mL} / \mathrm{min} / 1.73 \mathrm{~m}^{2}$ (both $P<0.05$ ). CysC/Cr showed no significant differences between the control and diabetic group or among the three diabetic subgroups.

Correlation analysis showed that Lg FABP4 significantly correlated with age, BMI, SBP, WC, and rGFR (all $P<0.05$, Table 3). After adjustment for covariates including sex, age, BMI, SBP, and WC, Lg FABP4 was independently associated with $\operatorname{HbAlc}(P=0.022)$ and $\operatorname{rGFR}(P<0.0001)$.

Multivariate stepwise linear regression analysis showed that UACR was associated with $\mathrm{CysC/Cr}$ and $\mathrm{RBP} 4$, while rGFR was associated with FABP4 and UACR (all $P<0.0001$, Table 4). After adjustment for sex, age, BMI, SBP, WC, HbAlc, and use of medications such as ARB and ACEI, only FABP4 was significantly associated with $\operatorname{rGFR}(P<0.0001)$, and UACR remained significantly associated with $\mathrm{CysC/Cr}$ and RBP4.

\section{Discussion}

In the present study, among individuals with different renal function status, we found that FABP4 levels were significantly higher in T2DM patients compared with healthy controls. Elevated FABP4 levels were independently associated with the reduction in $\mathrm{rGFR}$ in T2DM patients irrespective of UACR levels. CysC/Cr and RBP4 significantly correlated with UACR.

Previous studies have reported that increased FABP4 levels were associated with the deterioration in renal function in both humans and animals and therefore served as an independent indicator for the progression of nephropathy $[20,43,44]$. The mechanisms behind the elevation of FABP 4 in patients with diabetic kidney disease are not yet fully understood. It is known that FABP4 is abundantly expressed in adipocytes, macrophages, and endothelial cells [45-47]. Firstly, it is suggested that, during the early stage of DN, accumulation of active macrophages is more evident in the kidney because of the elevation in oxidative stress and chronic inflammation, which consequently induce increased 


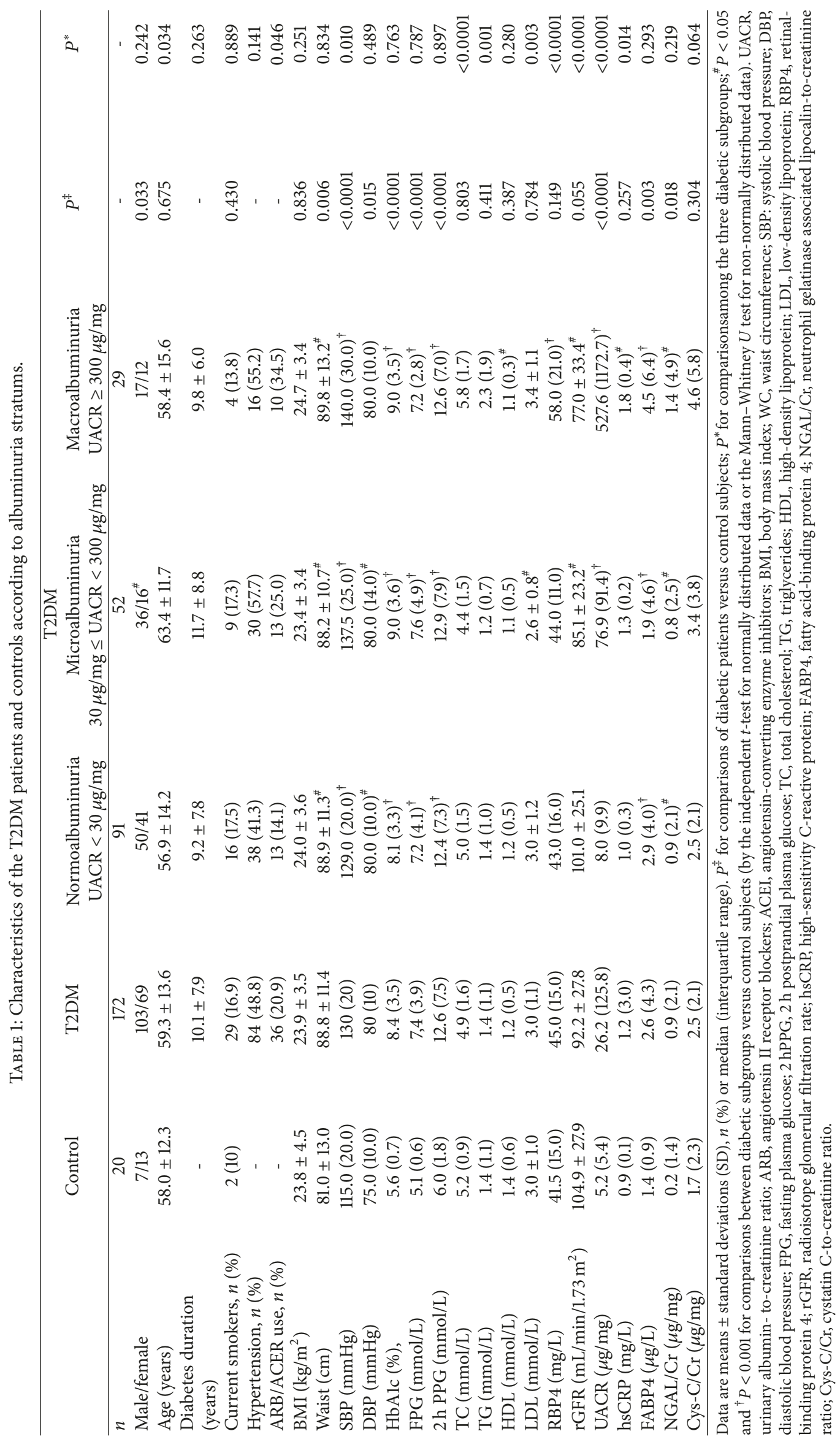




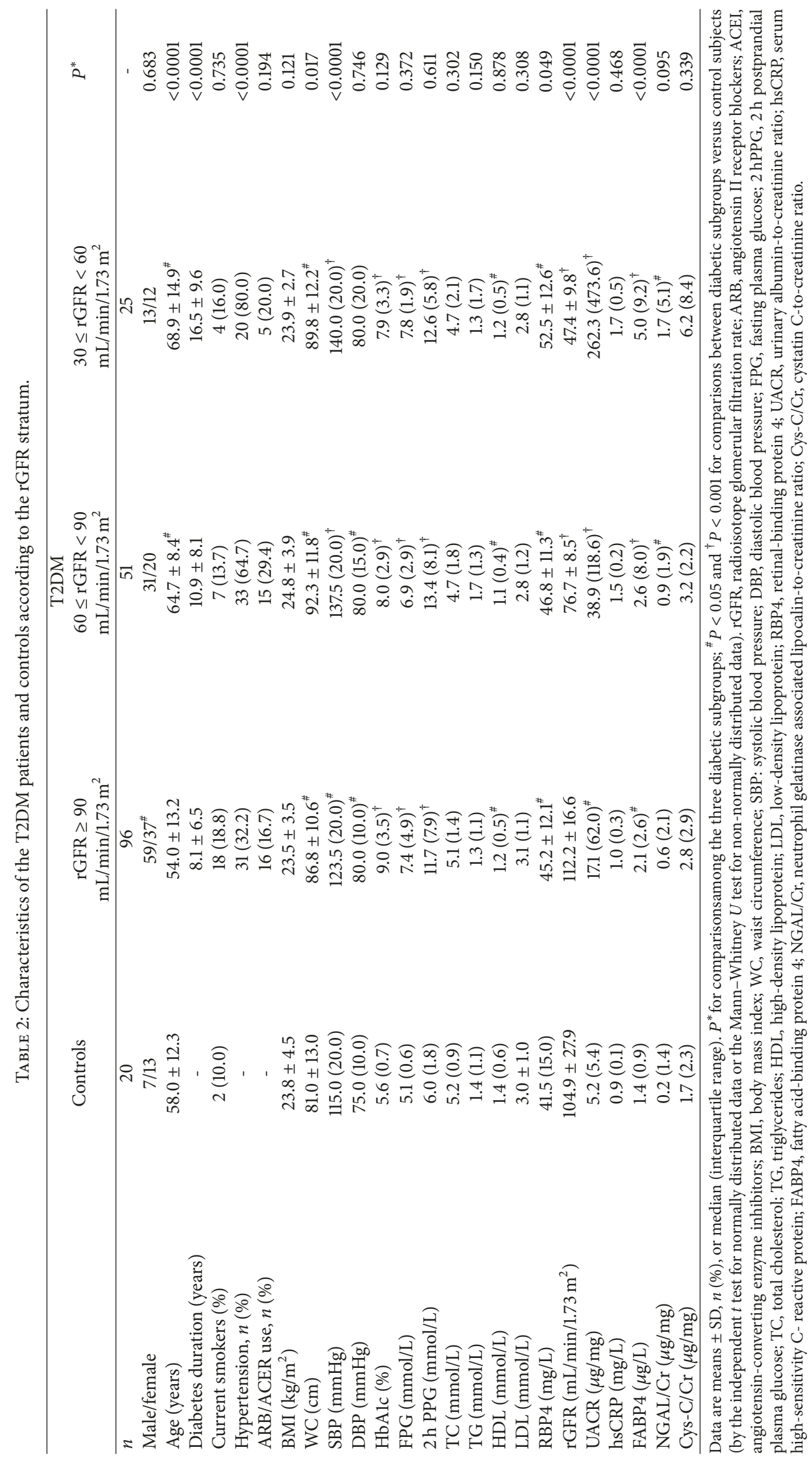


TABLE 3: Correlation analysis for Lg FABP in T2DM patients.

\begin{tabular}{|c|c|c|c|c|}
\hline & & & & \\
\hline & $r$ & $P$ & $r^{\S}$ & $P^{\S}$ \\
\hline $\operatorname{Sex}^{*}$ & 0.286 & $<0.0001$ & & \\
\hline Age (years) & 0.195 & 0.015 & & \\
\hline Diabetes duration (years) & 0.095 & 0.249 & & \\
\hline BMI $\left(\mathrm{kg} / \mathrm{m}^{2}\right)$ & 0.272 & 0.001 & & \\
\hline $\mathrm{WC}(\mathrm{cm})$ & 0.292 & $<0.0001$ & & \\
\hline $\mathrm{SBP}^{*}(\mathrm{mmHg})$ & 0.190 & 0.023 & & \\
\hline $\operatorname{HbAlc}(\%)^{\dagger}$ & 0.068 & 0.427 & 0.205 & 0.022 \\
\hline $\mathrm{TC}^{*}(\mathrm{mmol} / \mathrm{L})$ & 0.004 & 0.961 & -0.138 & 0.133 \\
\hline $\mathrm{TG}^{*}(\mathrm{mmol} / \mathrm{L})$ & 0.140 & 0.086 & 0.081 & 0.355 \\
\hline $\mathrm{HDL}^{*}(\mathrm{mmol} / \mathrm{L})$ & 0.092 & 0.260 & 0.132 & 0.129 \\
\hline $\mathrm{LDL}(\mathrm{mmol} / \mathrm{L})$ & -0.028 & 0.734 & 0.019 & 0.830 \\
\hline $\mathrm{RBP} 4(\mathrm{mg} / \mathrm{L})$ & 0.082 & 0.337 & 0.001 & 0.987 \\
\hline $\operatorname{rGFR}\left(\mathrm{mL} / \mathrm{min} / 1.73 \mathrm{~m}^{2}\right)$ & -0.350 & $<0.0001$ & -0.349 & $<0.0001$ \\
\hline hsCRP $(\mathrm{mg} / \mathrm{L})^{\dagger}$ & 0.148 & 0.083 & 0.166 & 0.162 \\
\hline $\mathrm{UACR}^{*}(\mu \mathrm{g} / \mathrm{mg})$ & 0.057 & 0.481 & 0.067 & 0.435 \\
\hline Cys-C/Cr $(\mu \mathrm{g} / \mathrm{mg})^{\dagger}$ & 0.009 & 0.915 & 0.042 & 0.633 \\
\hline $\mathrm{NGAL} / \mathrm{Cr}(\mu \mathrm{g} / \mathrm{mg})^{\dagger}$ & 0.134 & 0.097 & 0.082 & 0.340 \\
\hline
\end{tabular}

${ }^{\dagger} \log$-transformed variables; Pearson correlation analysis for normally distributed variables; ${ }^{*}$ Spearman correlation analysis for non-normally distributed variables; ' adjustment for sex, age, BMI, WC, and SBP used partial correlation analysis. FABP4, fatty acid-binding protein 4; BMI, body mass index; WC, waist circumference; SBP: systolic blood pressure; TC, total cholesterol; TG, triglycerides; HDL, high-density lipoprotein; LDL, low-density lipoprotein; RBP4, retinal-binding protein 4; rGFR, radioisotope glomerular filtration rate; hsCRP, high-sensitivity C-reactive protein; UACR, urinary albumin-to-creatinine ratio; Cys-C/Cr, cystatin C-to-creatinine ratio; $\mathrm{NGAL} / \mathrm{Cr}$, neutrophil gelatinase associated lipocalin-to-creatinine ratio.

TABLE 4: Multivariable regression analysis of various biomarkers versus UACR or rGFR.

\begin{tabular}{|c|c|c|c|c|}
\hline & \multicolumn{2}{|c|}{ UACR } & \multicolumn{2}{|c|}{ rGFR } \\
\hline & Standard $\beta$ & $P$ & Standard $\beta$ & $P$ \\
\hline \multicolumn{5}{|l|}{ Model 1} \\
\hline FABP4 & 0.124 & 0.109 & -0.364 & $<0.0001$ \\
\hline NGAL/Cr & 0.110 & 0.137 & -0.200 & 0.006 \\
\hline CysC/Cr & 0.307 & $<0.0001$ & -0.091 & 0.235 \\
\hline RBP4 & 0.440 & $<0.0001$ & -0.163 & 0.034 \\
\hline UACR & & & -0.250 & $<0.0001$ \\
\hline \multicolumn{5}{|l|}{ Model 2} \\
\hline FABP4 & 0.107 & 0.289 & -0.349 & $<0.0001$ \\
\hline NGAL/Cr & 0.065 & 0.455 & -0.095 & 0.220 \\
\hline CysC/Cr & 0.295 & 0.001 & -0.077 & 0.331 \\
\hline RBP4 & 0.441 & $<0.0001$ & -0.159 & 0.045 \\
\hline UACR & & & -0.232 & 0.001 \\
\hline \multicolumn{5}{|l|}{ Model 3} \\
\hline FABP4 & 0.063 & 0.488 & -0.367 & $<0.0001$ \\
\hline NGAL/Cr & 0.082 & 0.343 & -0.052 & 0.554 \\
\hline CysC/Cr & 0.450 & $<0.0001$ & -0.046 & 0.652 \\
\hline RBP4 & 0.519 & $<0.0001$ & -0.114 & 0.250 \\
\hline UACR & & & -0.118 & 0.235 \\
\hline
\end{tabular}

Model 1: crude model without covariate adjustment; model 2: adjustment for age, sex, BMI, waist circumference, SBP, HbAlc, and use of ACEI/ARBs; model 3: adjustment for age, sex, BMI, WC, SBP, use of ACEI/ARBs, and all the other potential damage biomarkers in the table. UACR, urinary albumin-to-creatinine ratio; rGFR, radioisotope glomerular filtration rate; FABP4, fatty acid-binding protein 4; NGAL/Cr, neutrophil gelatinase associated lipocalin-to-creatinine ratio; Cys-C/Cr, cystatin C-to-creatinine ratio; RBP4, retinal-binding protein 4. 
expression of serum FABP4 [12, 20]. Secondly, damage to glomeruli and tubulointerstitium might result in both decreased glomerular filtration and increased tubular reabsorption, leading to an increase in FABP4 in the circulation [44]. Thirdly, Okazaki et al. firstly reported that urinary excretion of FABP4 was associated with progression of proteinuria and renal dysfunction in healthy subjects [48]. The authors suggested that U-FABP4 reflects damage of glomerular with the hypothesis proposed by Tanaka et al. that main source of U-FABP4 is derived from ectopic expression of glomerular FABP4 rather than increased adiposity and that locally increased FABP4 in the glomerulus affects renal dysfunction [49]. However, there is no report about the relationship of U-FABP4 and renal dysfunction in patients with T2DM up to date.

Although UACR is the most widely used indicator for diabetic kidney damage and has been shown to predict the progression of chronic renal disease in patients with diabetes even with normal eGFR [50-52], there was a negative correlation between rGFR and UACR in the current study. Similar to our results, Cabré et al. also reported that FABP4, but not UACR, was independently associated with eGFR in T2DM patients with eGFR $\geq 60 \mathrm{~mL} / \mathrm{min} / 1.73 \mathrm{~m}^{2}$ [44]. However, it is unlikely that a single biomarker predicts the impairment of DN since the pathophysiological processes during the course of the disease are complex. Therefore, serum FABP4 along with UACR or a panel of biomarkers might be more sensitive for the detection of early DN.

In the pathogenesis and progression of $\mathrm{DN}$, both glomerular dysfunction and tubulointerstitial damage play crucial roles. As a tubular damage marker, either in plasma or in urine, NGAL is reported to increase in the early stage of $\mathrm{DN}$ and can be a predictor for kidney disease progression independent of GFR $[28,30]$. Results of previous studies are not conclusive, as Nauta et al. reported that urine NGAL was independently associated with UACR irrespective of eGFR [53], while Chou et al. reported that urine NGAL may not be a predictive factor associated with a decline in GFR in patients with T2DM [54]. In the current study, we found that urine NGAL/Cr did not have a significant correlation with either UACR or rGFR. It is thought that the tubulointerstitial injury in our T2DM patients with early renal impairment may be less severe, which might be the reason for the negative correlation between NGAL/Cr and UACR or rGFR. We also found that UACR significantly correlated with $\mathrm{CysC/Cr}$ and $\mathrm{RBP} 4$. This finding is consistent with previous studies which reported that both the two biomarkers were sensitive for the detection of DN in the early stage [55-57].

A strength of the current study is that rGFR was measured using the radio isotopic method, which allowed for the reliable and accurate measurement of renal function. We used urinary creatinine to correct NGAL and CysC to reduce biological variability [58].

This study had some limitations. First, the study was a single-center cross-sectional study with a small number of patients. Secondly, to confirm the role of FABP4 in the prediction of renal impairment in T2DM, a follow-up data regarding the change of rGFR in the higher FABP4 group and lower FABP4 group would be needed. However, the present study was only a cross-sectional study lacking a completed follow-up data. Thirdly, as a hospital-based study, most patients with T2DM enrolled in the study might have other macro- or microvascular complications, which may potentially affect the level of FABP4. Therefore, larger scale and multicenter prospective studies with long-term followup periods are required to validate the results.

\section{Conclusion}

Serum FABP4 had an inverse correlation with rGFR and could be an independent predictor for early DN. Greater reliability and a more sensitive analysis might be achieved by evaluating early diabetic renal function using UACR and a panel of biomarkers such as RBP4 and CysC/Cr in combination. Further prospective studies with long-term follow-up periods are needed to confirm the results.

\section{Disclosure}

Xiaoqing $\mathrm{Ni}$ and Yunjuan $\mathrm{Gu}$ are co-first authors of this article.

\section{Conflicts of Interest}

The authors declare that there are no conflicts of interest regarding the publication of this article.

\section{Authors' Contributions}

$\mathrm{X}$. Ni and Y. Gu contributed equally to this work.

\section{Acknowledgments}

This work was supported by grants from the National 973 Program (2011CB504001); the Six Top Talents Foundation of Jiangsu Province, China (2016-WSN-098); the Natural Science Foundation of Nantong (HS2014036 and MS22015126); the Open Research Project of Shanghai Key Laboratory of Diabetes Mellitus (SHKLD-KF-1303); the Jiangsu Health and Family Planning Commission (Y2015070); and the New Clinical Project of the Affiliated Hospital of Nantong University (2016-047). The authors thank Dr. Huang Zhimin for helping them with the language in the manuscript and all the participants of the study. They thank Alexander Pishief, LLB, BBmedSc, from Liwen Bianji, Edanz Group China (http://www.liwenbianji.cn/ac), for editing the English text of a draft of this manuscript.

\section{References}

[1] J. L. Gross, M. J. de Azevedo, S. P. Silveiro, L. H. Canani, M. L. Caramori, and T. Zelmanovitz, "Diabetic nephropathy: diagnosis, prevention, and treatment," Diabetes Care, vol. 28, no. 1, pp. 164-176, 2005

[2] National Kidney Foundation, "K/DOQI clinical practice guidelines for chronic kidney disease: evaluation, classification, and 
stratification," American Journal of Kidney Diseases, vol. 39, supplement 1, no. 2, pp. S1-266, 2002.

[3] D. E. Weiner and M. J. Sarnak, "A decade after the KDOQI CKD guidelines: impact on the cardiovascular disease-CKD paradigm," American Journal of Kidney Diseases, vol. 60, no. 5, pp. 710-712, 2012.

[4] Y. Slinin, A. Ishani, T. Rector et al., "Management of hyperglycemia, dyslipidemia, and albuminuria in patients with diabetes and CKD: a systematic review for a KDOQI clinical practice guideline," American Journal of Kidney Diseases, vol. 60, no. 5, pp. 747-769, 2012.

[5] M.-J. McLaughlin and A. E. Courtney, "Early recognition of CKD can delay progression," The Practitioner, vol. 257, no. 1758, pp. 13-17, 2013.

[6] Kidney Disease and Improving Global Outcomes (KDIGO) CKD Work Group, "KDIGO 2012 clinical practice guideline for the evaluation and management of chronic kidney disease," Kidney International Supplements, vol. 3, pp. 1-150, 2013.

[7] A. S. Levey, "A decade after the KDOQI CKD guidelines," American Journal of Kidney Diseases, vol. 60, no. 5, pp. 683-685, 2012.

[8] A. S. Levey, C. Becker, and L. A. Inker, "Glomerular filtration rate and albuminuria for detection and staging of acute and chronic kidney disease in adults: a systematic review," The Journal of the American Medical Association, vol. 313, no. 8, pp. 837-846, 2015.

[9] J. S. Fleming, M. A. Zivanovic, G. M. Blake, M. Burniston, and P. S. Cosgriff, "Guidelines for the measurement of glomerular filtration rate using plasma sampling," Nuclear Medicine Communications, vol. 25, no. 8, pp. 759-769, 2004.

[10] K. Matsushita, M. van der Velde, and B. C. Astor, "Association of estimated glomerular filtration rate and albuminuria with all-cause and cardiovascular mortality in general population cohorts: a collaborative meta-analysis," The Lancet, vol. 375, pp. 2073-2081, 2010.

[11] M. Kravaritou, A. Thanopoulou, B. Karamanos et al., "Evidence that even "normal" albuminuria may denote incipient GFR reduction in patients with type 2 diabetes mellitus," Diabetes Research and Clinical Practice, vol. 85, no. 3, pp. 317-321, 2009.

[12] A. Cabré, I. Lázaro, J. Girona et al., "Fatty acid binding protein 4 is increased in metabolic syndrome and with thiazolidinedione treatment in diabetic patients," Atherosclerosis, vol. 195, no. 1, pp. e150-e158, 2007.

[13] G. Tuncman, E. Erbay, X. Hom et al., "A genetic variant at the fatty acid-binding protein aP2 locus reduces the risk for hypertriglyceridemia, type 2 diabetes, and cardiovascular disease," Proceedings of the National Acadamy of Sciences of the United States of America, vol. 103, no. 18, pp. 6970-6975, 2006.

[14] D. Yeung, A. Xu, C. Cheung et al., "Serum Adipocyte Fatty Acid-Binding Protein Levels Were Independently Associated With Carotid Atherosclerosis," Arteriosclerosis, Thrombosis, and Vascular Biology, vol. 27, no. 8, pp. 1796-1802, 2007.

[15] J. B. Boord, K. Maeda, L. Makowski et al., "Combined adipocyte-macrophage fatty acid-binding protein deficiency improves metabolism, atherosclerosis, and survival in apolipoprotein E-deficient mice," Circulation, vol. 110, no. 11, pp. 1492-1498, 2004.

[16] L. Makowski, J. B. Boord, K. Maeda et al., "Lack of macrophage fatty-acid-binding protein aP2 protects mice deficient in apolipoprotein E against atherosclerosis," Nature Medicine, vol. 7, no. 6, pp. 699-705, 2001.
[17] A. Xu, A. W. K. Tso, B. M. Y. Cheung et al., "Circulating adipocyte-fatty acid binding protein levels predict the development of the metabolic syndrome: a 5-year prospective study," Circulation, vol. 115, no. 12, pp. 1537-1543, 2007.

[18] G. Sommer, M. Ziegelmeier, A. Bachmann et al., "Serum levels of adipocyte fatty acid-binding protein (AFABP) are increased in chronic haemodialysis (CD)," Clinical Endocrinology, vol. 69, no. 6, pp. 901-905, 2008.

[19] F. Toruner, A. E. Altinova, M. Akturk et al., "The relationship between adipocyte fatty acid binding protein-4, retinol binding protein-4 levels and early diabetic nephropathy in patients with type 2 diabetes," Diabetes Research and Clinical Practice, vol. 91, no. 2, pp. 203-207, 2011.

[20] D. C. Y. Yeung, A. Xu, A. W. K. Tso et al., "Circulating levels of adipocyte and epidermal fatty acid-binding proteins in relation to nephropathy staging and macrovascular complications in type 2 diabetic patients," Diabetes Care, vol. 32, no. 1, pp. 132134, 2009.

[21] Q. Yang, T. E. Graham, N. Mody et al., "Serum retinol binding protein 4 contributes to insulin resistance in obesity and type 2 diabetes," Nature, vol. 436, no. 7049, pp. 356-362, 2005.

[22] J. Janke, S. Engeli, M. Boschmann et al., "Retinol-binding protein 4 in human obesity," Diabetes, vol. 55, no. 10, pp. 28052810, 2006.

[23] T. E. Graham, Q. Yang, M. Blüher et al., "Retinol-binding protein 4 and insulin resistance in lean, obese, and diabetic subjects," The New England Journal of Medicine, vol. 354, no. 24, pp. 2552-2563, 2006.

[24] A. Cabré, I. Lázaro, J. Girona et al., "Retinol-binding protein 4 as a plasma biomarker of renal dysfunction and cardiovascular disease in Type 2 diabetes," Journal of Internal Medicine, vol. 262, no. 4, pp. 496-503, 2007.

[25] M. Murata, T. Saito, T. Otani et al., "An increase in serum retinol-binding protein 4 in the type 2 diabetic subjects with nephropathy," Endocrine Journal, vol. 56, no. 2, pp. 287-294, 2009.

[26] R. N. Moresco, M. B. Sangoi, J. A. M. De Carvalho, E. Tatsch, and G. V. Bochi, "Diabetic nephropathy: traditional to proteomic markers," Clinica Chimica Acta, vol. 421, pp. 17-30, 2013.

[27] K. Mori and K. Nakao, "Neutrophil gelatinase-associated lipocalin as the real-time indicator of active kidney damage," Kidney International, vol. 71, no. 10, pp. 967-970, 2007.

[28] S. E. Nielsen, H. Reinhard, D. Zdunek et al., "Tubular markers are associated with decline in kidney function in proteinuric type 2 diabetic patients," Diabetes Research and Clinical Practice, vol. 97, no. 1, pp. 71-76, 2012.

[29] W. Fu, S. Xiong, Y. Fang et al., "Urinary tubular biomarkers in short-term type 2 diabetes mellitus patients: a cross-sectional study," Endocrine Journal, vol. 41, no. 1, pp. 82-88, 2012.

[30] M. H. Mahfouz, A. M. Assiri, and M. H. Mukhtar, "Assessment of neutrophil gelatinase-associated lipocalin (NGAL) and retinol-binding protein 4 (RBP4) in type 2 diabetic patients with nephropathy," Biomarker Insights, vol. 11, pp. 31-40, 2016.

[31] M. Abrahamson, I. Olafsson, A. Palsdottir et al., "Structure and expression of the human cystatin C gene," Biochemical Journal, vol. 268, no. 2, pp. 287-294, 1990.

[32] L. D. Dworkin, "Serum cystatin C as a marker of glomerular filtration rate," Current Opinion in Nephrology and Hypertension, vol. 10, no. 5, pp. 551-553, 2001.

[33] L. A. Stevens, J. Coresh, C. H. Schmid et al., "Estimating GFR using serum cystatin $\mathrm{C}$ alone and in combination with serum 
creatinine: a pooled analysis of 3,418 individuals with CKD," American Journal of Kidney Diseases, vol. 51, no. 3, pp. 395-406, 2008.

[34] S. K. Swan, "The search continues-an ideal marker of GFR," Clinical Chemistry, vol. 43, no. 6, pp. 913-914, 1997.

[35] L. M. Russo, R. M. Sandoval, S. B. Campos, B. A. Molitoris, W. D. Comper, and D. Brown, "Impaired tubular uptake explains albuminuria in early diabetic nephropathy," Journal of the American Society of Nephrology, vol. 20, no. 3, pp. 489-494, 2009.

[36] E. Randers and E. J. Erlandsen, "Serum cystatin C as an endogenous marker of the renal function-a review," Clinical Chemistry and Laboratory Medicine, vol. 37, no. 4, pp. 389-395, 1999.

[37] R. Hojs, S. Bevc, R. Ekart, M. Gorenjak, and L. Puklavec, "Serum cystatin $\mathrm{C}$ as an endogenous marker of renal function in patients with mild to moderate impairment of kidney function," Nephrology Dialysis Transplantation, vol. 21, no. 7, pp. 18551862, 2006.

[38] M. Madero, M. J. Sarnak, and L. A. Stevens, "Serum cystatin C as a marker of glomerular filtration rate," Current Opinion in Nephrology and Hypertension, vol. 15, no. 6, pp. 610-616, 2006.

[39] S. W. L. Pinto, R. Sesso, E. Vasconcelos, Y. J. Watanabe, and A. M. Pansute, "Cystatin C is not more sensitive than creatinine for detecting early renal impairment in patients with diabetes," American Journal of Kidney Diseases, vol. 38, no. 2, pp. 310-316, 2001.

[40] M. Sokolski, R. Zymliński, J. Biegus et al., "Urinary levels of novel kidney biomarkers and risk of true worsening renal function and mortality in patients with acute heart failure," European Journal of Heart Failure, vol. 19, no. 6, pp. 760-767, 2017.

[41] J. Helmersson-Karlqvist, J. Ärnlöv, A. C. Carlsson, L. Lind, and A. Larsson, "Urinary KIM-1, but not urinary cystatin C, should be corrected for urinary creatinine," Clinical Biochemistry, vol. 49, no. 15, pp. 1164-1166, 2016.

[42] K. G. M. M. Alberti and P. Z. Zimmet, "Definition, diagnosis and classification of diabetes mellitus and its complications. Part 1: diagnosis and classification of diabetes mellitus. Provisional report of a WHO consultation," Diabetic Medicine, vol. 15, no. 7, pp. 539-553, 1998.

[43] T. Ebert, L. M. Hopf, U. Wurst et al., "Circulating adipocyte fatty acid binding protein is increased in chronic and acute renal dysfunction," Nutrition, Metabolism and Cardiovascular Diseases, vol. 24, no. 9, pp. 1027-1034, 2014.

[44] A. Cabré, I. Lázaro, J. Girona et al., "Plasma fatty acid-binding protein 4 increases with renal dysfunction in type 2 diabetic patients without microalbuminuria," Clinical Chemistry, vol. 54, no. 1, pp. 181-187, 2008.

[45] N. Ribarik Coe and D. A. Bernlohr, "Physiological properties and functions of intracellular fatty acid- binding proteins," Biochimica et Biophysica Acta (BBA) - Lipids and Lipid Metabolism, vol. 1391, no. 3, pp. 287-306, 1998.

[46] H. Elmasri, C. Karaaslan, Y. Teper et al., "Fatty acid binding protein 4 is a target of VEGF and a regulator of cell proliferation in endothelial cells," The FASEB Journal, vol. 23, no. 11, pp. 38653873, 2009.

[47] Y. Fu, L. Luo, N. Luo, and W. Timothy Garvey, "Lipid metabolism mediated by adipocyte lipid binding protein (ALBP/aP2) gene expression in human THP-1 macrophages," Atherosclerosis, vol. 188, no. 1, pp. 102-111, 2006.
[48] Y. Okazaki, M. Furuhashi, M. Tanaka et al., "Urinary excretion of fatty acid-binding protein 4 is associated with albuminuria and renal dysfunction," PLoS ONE, vol. 9, no. 12, Article ID e115429, 2014.

[49] M. Tanaka, M. Furuhashi, Y. Okazaki et al., "Ectopic expression of fatty acid-binding protein 4 in the glomerulus is associated with proteinuria and renal dysfunction," Nephron Clinical Practice, vol. 128, pp. 345-351, 2014.

[50] H. J. Lambers Heerspink, J. W. Brinkman, S. J. L. Bakker, R. T. Gansevoort, and D. De Zeeuw, "Update on microalbuminuria as a biomarker in renal and cardiovascular disease," Current Opinion in Nephrology and Hypertension, vol. 15, no. 6, pp. 631636, 2006.

[51] P. Ochodnicky, S. Vettoretti, R. H. Henning, H. Buikema, and P. E. Van Dokkum, "Endothelial dysfunction in chronic kidney disease: Determinant of susceptibility to end-organ damage and therapeutic response," Journal of Nephrology, vol. 19, no. 3, pp. 246-258, 2006.

[52] K. E. Larmour, A. P. Maxwell, and A. E. Courtney, "Improving early detection of chronic kidney disease," The Practitioner, vol. 259, no. 1779, pp. 19-23, 2015.

[53] F. L. Nauta, W. E. Boertien, S. J. L. Bakker et al., "Glomerular and tubular damage markers are elevated in patients with diabetes," Diabetes Care, vol. 34, no. 4, pp. 975-981, 2011.

[54] K.-M. Chou, C.-C. Lee, C.-H. Chen, and C.-Y. Sun, "Clinical value of NGAL, L-FABP and albuminuria in predicting GFR decline in type 2 diabetes mellitus patients," PLoS ONE, vol. 8, no. 1, article e54863, 2013.

[55] H. Chen and H. Li, "Clinical Implication of Cystatin C and 32 -Microglobulin in Early Detection of Diabetic Nephropathy," Clinical Laboratory, vol. 63, no. 02, pp. 241-247, 2017.

[56] C. Y. Hong and K. S. Chia, "Markers of diabetic nephropathy," Journal of Diabetes and its Complications, vol. 12, no. 1, pp. 4360, 1998.

[57] S. M. Titan, J. M. Vieira Jr., W. V. Dominguez et al., "Urinary MCP-1 and RBP: independent predictors of renal outcome in macroalbuminuric diabetic nephropathy," Journal of Diabetes and its Complications, vol. 26, no. 6, pp. 546-553, 2012.

[58] J. Helmersson-Karlqvist, J. Ärnlöv, and A. Larsson, "Day-today variation of urinary NGAL and rational for creatinine correction," Clinical Biochemistry, vol. 46, no. 1-2, pp. 70-72, 2013. 


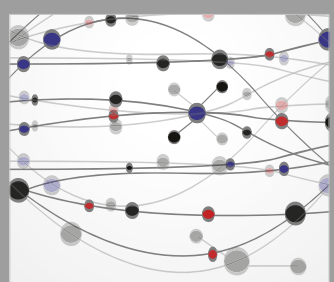

The Scientific World Journal
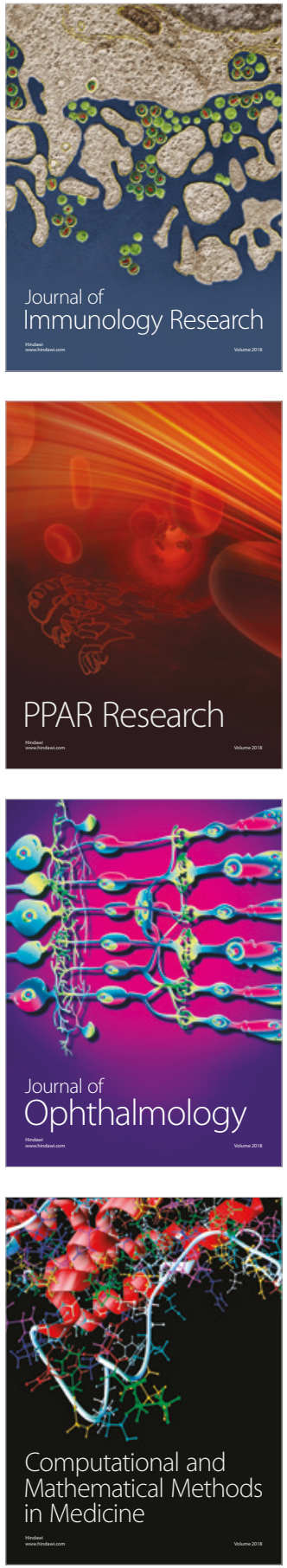

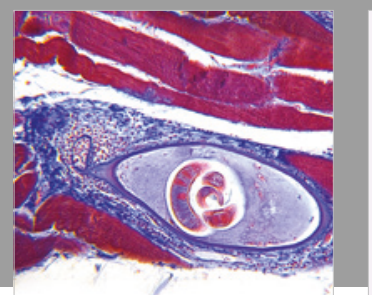

Gastroenterology Research and Practice

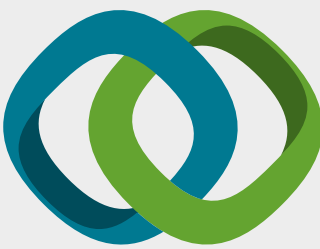

\section{Hindawi}

Submit your manuscripts at

www.hindawi.com
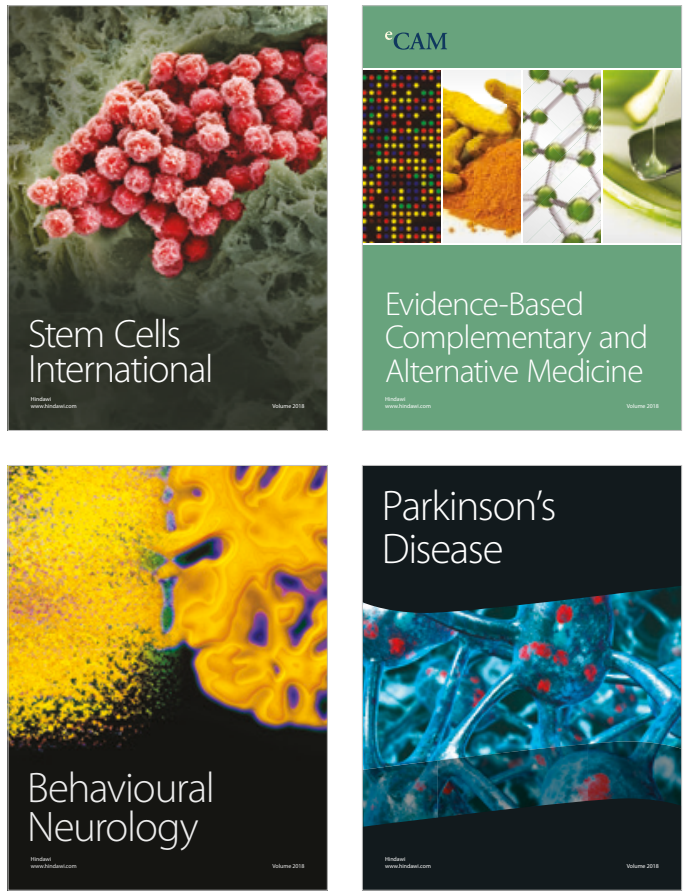

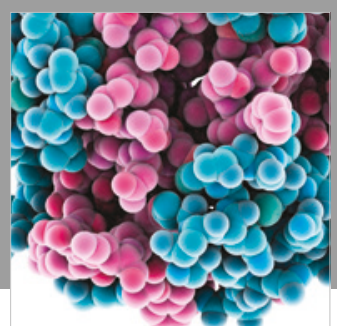

ournal of

Diabetes Research

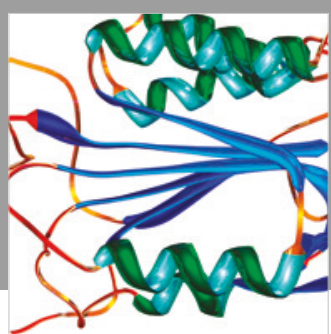

Disease Markers
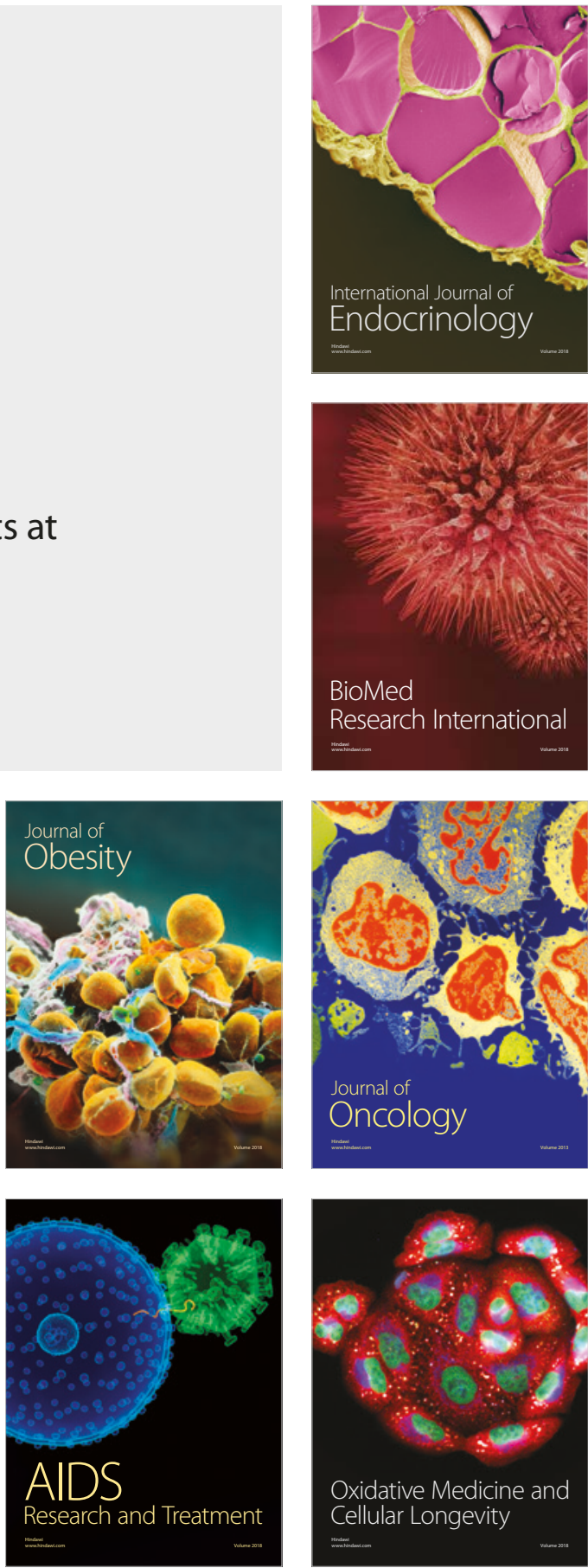